\title{
KHẢO SÁT ĐỘ CHÍNH XÁC CỦA CÔNG NGHỆ GNSS-RTK TRONG MỘT SỐ DẠNG CÔNG TÁC TRẮC ĐỊA CÔNG TRÌNH
}

\author{
TRÀ̀ VIẾT TUẤN ${ }^{(1)}$, DIÊM CÔNG TRANG(2) \\ ${ }^{\left({ }^{()} T r u o ̛ ̀ n g ~ Đ a ̣ i ~ h o ̣ c ~ M o ̉ ~-~ Đ i ̣ a ~ c h a ̂ ́ t ~ H a ̀ ~ N o ̣ ̂ i ~\right.}$ \\ (2) Viện Khoa học Công nghệ Xây dụng
}

\section{Tóm tắt:}

Nội dung của bài báo trình bày về kết quả nghiên cưu khảo sát độ chính xác đạt được của công nghệ GNSS-RTK khi định vị điểm đon trong điều kiện Việt Nam. Co sở lý thuyết của phuong pháp đo đạc khảo sát thực nghiệm, kết quả đo đạc thực nghiệm và đánh giá khả năng úng dụng của công nghệ tiên tiến này trong một số dạng công tác trắc địa công trình ở Việt Nam.

\section{1. Đặt vấn đề}

Hiện nay trong công tác trắc địa công trình có nhiều dạng công việc đòi hỏi phải xác định được toạ độ tức thời của một số điểm đặc biệt trền công trình theo thời gian thực nhằm mục đích thực hiện các dạng công tác chuyên môn phục vụ thi công xây dựng hoặc đánh giá độ ổn định của công trình. Có thể lấy một số ví dụ điển hình sau đây:

- Trong công tác trắc địa thi công xây dựng nhà siêu cao tầng: cần phải quan trắc xác định sự dao động của toà nhà trong quá trình thi công do tác động ảnh hưởng của các yếu tố môi trường bên ngoài [3].

- Quan trắc sự dao động của cầu dây văng khi thi công xây dựng hoặc trong quá trình khai thác sử dụng.

- Quan trắc và cảnh báo các hiện tượng trượt lở của các khu vực đồi núi phía Bắc hay tại các tuyến sông rạch vùng đồng bằng sông Cửu Long.

Vấn đề cần đặt ra nghiên cứu ở đây là: Cần phải lựa chọn công nghệ, thiết bị hiện đại đang có tại Việt Nam có độ chính xác đáp ứng được các yêu cầu cần thiết đối với từng dạng công trình cụ thể đang được thi công xây dựng hoặc đưa vào khai thác sử dụng. Vì vậy mà cần phải nghiên cứu lựa chọn công nghệ mới cho phép xác định liên tục vị trí điểm quan sát theo thời gian thực và cần phải khảo sát độ chính xác đạt được của dạng công nghệ GNSS-RTK trong điều kiện Việt Nam

\section{Cơ sở lý thuyết và phương pháp nghiên cứu}

\subsection{Công nghệ GNSS-RTK}

GNSS-RTK là kỹ thuật định vị tương đối động. Khi đó người ta sử dụng một máy thu đặt cố định tại điểm đã biết toạ độ và độ cao (điểm $\mathrm{P}_{1}$ ), gọi là trạm cơ sở, còn máy thu thứ hai cho phép di chuyển trong khi đo, gọi là trạm động (điểm $\mathrm{P}_{2}$ - hình 1). Trong phương pháp đo này, thời gian thu tín hiệu tại mỗi điểm đo được rút ngắn chỉ còn từ một vài giây đến vài phút. Khi áp dụng kỹ thuật định vị tương đối động xử lý tức thời RTK, cần phải truyền ngay số liệu đo khoảng cách giả, trị đo pha sóng tải tại trạm cơ sở tới trạm động bằng thiết bi kết nối số liệu không dây (Radio link) hoặc sóng 3G.

Tại trạm động việc giải số nguyên đa trị được thực hiện để xác định tức thời véc tơ cạnh [1]. Như vậy dựa vào công nghệ đo GNSS-RTK ta có thể xác định được liên tục toạ độ và độ cao của trạm động theo thời gian thực và tần suất đo phụ thuộc vào tần suất thu tín hiệu của thiết bị GNSS được sử dụng.

Hiện nay trong lĩnh vực trắc địa công trình có rất nhiều dạng công việc cần sư dụng đến công nghệ xác định toạ độ điểm động theo thời gian thực:

- Đo đạc xác định vị trí điểm cơ sở trên các sàn thi công tại thời điểm $\mathrm{t}_{\mathrm{i}}$ trong thi công xây dựng nhà siêu cao tầng bằng công nghệ GNSSRTK;

- Ghép nối trực tiếp hệ GNSS-RTK vào hệ thống máy đo sâu hồi âm dùng trong đo vẽ bản đồ địa hình dưới nước tỷ lệ lớn;

Ngày nhận bài: 03/9/2018, ngày chuyển phản biện: 05/9/2018, ngày chấp nhận phản biện: 18/9/2018, ngày chấp nhận đăng: 20/9/2018 


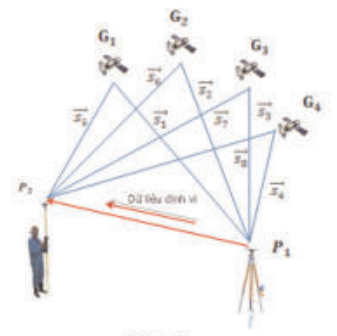

Hinh 1

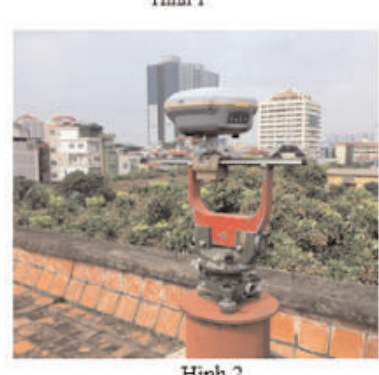

Hinh 2

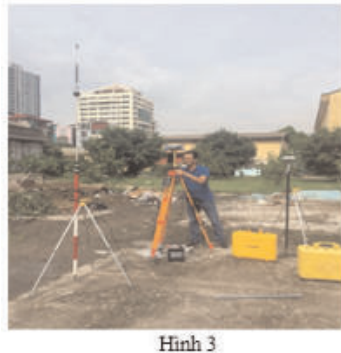

- Sử dụng công nghệ GNSS-RTK trong quan trắc các hiện tượng trượt lở tại các ta luy dương và âm trong thi công xây dựng các tuyến đường ở vùng núi phía Bắc của nước ta .V.v...

Độ chính xác của công nghệ GNSS-RTK khi sử dụng máy thu GNSS R8s theo khuyến cáo của hãng sản xuất [4] đạt:

$$
\begin{array}{lll}
\text { + Mặt bằng } & : \pm 8 \mathrm{~mm}+1 \mathrm{ppm} & \text { RMS } \\
\text { + Độ cao } & : \pm 15 \mathrm{~mm}+1 \mathrm{ppm} & \text { RMS }
\end{array}
$$

Để có thể nâng cao được hiệu quả ứng dụng công nghệ GNSS-RTK trong trắc địa công trình thì cần phải khảo sát đánh giá độ chính xác đạt được thực tế của công nghệ GNSS-RTK trong điều kiện đo đạc thực tế ở Việt Nam.

\subsection{Khảo sát độ chính xác của công nghệ} GNSS-RTK khi xác định tọ̣ độ điểm quan sát theo thơ gian thục

Chúng tôi đã tiến hành đo đạc khảo sát độ chính xác định vị điểm bằng công nghệ GNSS-
RTK tại khu vực Viện Khoa học - Công nghệ Xây dựng vào ngày 3/8/2018 với hệ thống máy thu của hãng Trimble: GNSS R8s (hình 2). Tại trạm Base đặt máy thu cố định trên một điểm mốc $\mathrm{A}$ đã biết toạ độ và độ cao (hình 3), tín hiệu cải chính được truyền từ trạm base đến trạm rove bằng sóng $3 \mathrm{G}$. Trạm rove $(\mathrm{CT}-1)$ được bố trí trên mái nhà tầng 3 của Viện Khoa học - Công nghệ Xây dựng (IBCT). Máy thu R8s được gắn trên một thiết bị chuyên dụng: đó là một hệ thống thước chuẩn bằng thép không gỉ, trên có khắc vạch chia đến $\mathrm{mm}$, được trang bị kính hiển vi đọc số đến $0.1 \mathrm{~mm}$. Máy thu GNSS có thể chuyển động trên thước với khoảng cách tuỳ ý, và giá trị chuyển dịch được đọc trực tiếp trên thước với độ chính xác đọc số $\pm 0.1 \mathrm{~mm}$ (hình 2).

- Đầu tiên chúng tôi đặt máy thu GNSS tại hai trạm (base và rove) và tiến hành thu tín hiệu trong thời gian khoảng 30 phút.

- Tiếp theo dịch chuyển máy thu GNSS trên thước đi từng đoạn $5 \mathrm{~mm}$ tại mỗi vị trí thu tín hiệu vệ tinh khoảng 1 đến 5 phút với tần suất thu tín hiệu là $1 \mathrm{~s}$.

- Tiến hành xử lý kết quả đo thực nghiệm bằng cách so sánh giữa chiều dài cạnh $\mathrm{S}_{\mathrm{i}}$ đo bằng công nghệ GNSS-RTK và khoảng cách dịch chuyển thực tế để đánh giá độ chính xác định vị của công nghệ GNSS-RTK theo các công thức sau đây:

1. Tại mỗi vị trí thu tín hiệu GNSS-RTK (thứ i) tính trị trung bình của $\mathrm{k}$ trị đo GNSS-RTK

$$
\begin{aligned}
X_{i} & =\frac{1}{k} \sum_{j=1}^{j-k} x_{j} \\
Y_{i} & =\frac{1}{k} \sum_{j=1}^{j-k} y_{j}
\end{aligned}
$$

2. Tính chiều dài mỗi đoạn đo theo công thức

$$
\text { Trong đó : } \begin{aligned}
& \mathrm{S}_{\mathrm{i}}=\sqrt{\Delta_{\mathrm{X}_{\mathrm{i}}}^{2}+\Delta_{\mathrm{Y}_{\mathrm{i}}}^{2}} \\
& \Delta_{\mathrm{X}_{\mathrm{i}}}=\mathrm{X}_{\mathrm{i}}-\mathrm{X}_{\mathrm{i}-1} \\
& \Delta_{\mathrm{Y}_{\mathrm{i}}}=\mathrm{Y}_{\mathrm{i}}-\mathrm{Y}_{\mathrm{i}-1}
\end{aligned}
$$

3. Tính độ lệch giữa trị đo GNSS-RTK và trị thực theo công thức

$\Delta_{\mathrm{i}}=\mathrm{S}_{\mathrm{i}}-\mathrm{S}_{\text {thvc }}$ 
Với $\mathrm{S}_{\text {thuc }}$ là chiều dài đoạn thước chuyển dịch (trong thực nghiệm này $\mathrm{S}_{\text {thuc }}=5 \mathrm{~mm}$ )

4. Đánh giá độ chính xác định vị của công nghệ GNSS-RTK bằng công thức đánh giá độ chính xác theo sai số thực [2]:

$$
\mathrm{m}= \pm \sqrt{\frac{\Delta \Delta}{\mathrm{n}}}
$$

Trong đó: $\Delta_{\mathrm{i}}$ là độ lệch của của đoạn đo bằng GNSS-RTK và giá trị thực của đoạn đo tính theo (6).

n là số đoạn đo kiểm tra trên thực địa.

\section{Kết quả đo đạc và tính toán thực nghiệm}

3.1. Kết quả đo thực nghiệm bằng công nghệ GNSS-RTK tại 9 vị trí thu tín hiệu được trình bày tóm tắt trong bảng 1 .

3.2. Tiến hành xử lý số liệu đo GNSS-RTK theo các công thức (1) đến (6) ta có kết quả như bảng 2 .

Trong bảng 2: Giá trị $\Delta_{\mathrm{i}}$ tính theo công thức (6) với $\mathrm{S}_{\text {thuc }}=0.005 \mathrm{~m}$.

3.3. Đánh giá độ chính xác định vị bằng công nghệ GNSS-RTK theo công thức (7) ta có:

$$
\mathrm{m}= \pm 0.00041(\mathrm{~m})
$$

hay độ chính xác định vị bằng công nghệ GNSS-RTK đạt độ chính xác:

$$
\mathrm{m}= \pm 0.41 \mathrm{~mm}
$$

Từ kết quả đo đạc và tính toán thực nghiệm cho thấy: độ chính xác định vị bằng công nghệ GNSS-RTK hoàn toàn có thể đáp ứng được các yêu cầu kỹ thuật cần thiết trong một số dạng công tác trắc địa công trình yêu cầu độ chính xác cao.

\section{Kết luận và kiến nghị}

- Có thể sử dụng công nghệ GNSS- RTK để xác định toạ độ tức thời của các điểm khống chế cơ sở trên các sàn thi công xây dựng nhà siêu cao tầng và trong một số dạng công tác trắc địa công trình yêu cầu độ chính xác cao bằng cách xác định toạ độ tức thời của điểm quan sát.

- Cần tiếp tục nghiên cứu các giải pháp kỹ thuật nhằm nâng cao độ chính xác và khả năng ứng dụng của công nghệ GNSS-RTK trong điều kiện sát với thực tế thi công xây dựng các công

\begin{tabular}{|c|c|c|c|c|c|c|}
\hline $\mathrm{i}$ & $\mathrm{j}$ & $\mathrm{X}_{\mathrm{i}}(\mathrm{m})$ & $\mathrm{Y}_{\mathrm{i}}(\mathrm{m})$ & $\mathrm{H}_{\mathrm{i}}(\mathrm{m})$ & & $\mathrm{t}_{\mathrm{i}}(\mathrm{s})$ \\
\hline \multirow{5}{*}{1} & 1 & 2328559.3050 & 581817.977 & 0.652 & IBST-CT1 & 2018Y08M03D09H45M45S \\
\hline & 2 & 2328559.3070 & 581817.980 & 0.656 & IBST-CT1 & 2018Y08M03D09H45M46S \\
\hline & $\ldots .$. & 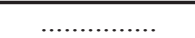 & ………....... & …….... & …........ & $2 \ldots \ldots \ldots$ \\
\hline & 22 & 2328559.3070 & 581817.976 & 0.650 & IBST-CT1 & 2018Y08M03D09H46M08S \\
\hline & 23 & 2328559.3060 & 581817.975 & 0.643 & IBST-CT1 & 2018Y08M03D09H46M09S \\
\hline \multirow{5}{*}{2} & 1 & 2328559.311 & 581817.974 & 0.648 & IBST-CT1 & 2018Y08M03D09H46M10S \\
\hline & 2 & 2328559.312 & 581817.974 & 0.652 & IBST-CT1 & 2018Y08M03D09H46M11S \\
\hline & $\ldots .$. & ………....... & ………....... & ............ & …........ & …………........ \\
\hline & 25 & 2328559.311 & 581817.976 & 0.647 & IBST-CT1 & 2018Y08M03D09H46M39S \\
\hline & 26 & 2328559.311 & 581817.975 & 0.643 & IBST-CT1 & 2018Y08M03D09H46M40S \\
\hline \multirow{5}{*}{3} & 1 & 2328559.314 & 581817.977 & 0.640 & IBST-CT1 & 2018Y08M03D09H46M41S \\
\hline & 2 & 2328559.315 & 581817.978 & 0.643 & IBST-CT1 & 2018Y08M03D09H46M42S \\
\hline & $\ldots .$. & ………....... & ………...... & …........ & …....... & …………........ \\
\hline & 30 & 2328559.318 & 581817.976 & 0.644 & IBST-CT1 & 2018Y08M03D09H47M10S \\
\hline & 31 & 2328559.316 & 581817.977 & 0.644 & IBST-CT1 & 2018Y08M03D09H47M11S \\
\hline$\ldots$ & $\ldots .$. & $\ldots$. & ....... & ............ & ……..... & ......... \\
\hline \multirow{5}{*}{9} & 1 & 2328559.345 & 581817.974 & 0.640 & IBST-CT1 & 2018Y08M03D09H48M32S \\
\hline & 2 & 2328559.346 & 581817.971 & 0.641 & IBST-CT1 & 2018Y08M03D09H48M33S \\
\hline & $\ldots . .$. & .......... & ………...... & …........ & …........ & …………....... \\
\hline & 7 & 2328559.347 & 581817.972 & 0.647 & IBST-CT1 & 2018Y08M03D09H48M38S \\
\hline & 8 & 2328559.347 & 581817.974 & 0.650 & IBST-CT1 & 2018Y08M03D09H48M39S \\
\hline
\end{tabular}
trình nhà siêu cao tầng ở nước ta. $\mathrm{O}$

Bảng 1: Kết quả đo GNSS-RTK tại các vị trí thu tín hiệu 
Bảng 2: Kết quả xủ lý số liệu đo GNSS-RTK

\begin{tabular}{|c|c|c|c|c|c|c|}
\hline $\mathrm{i}$ & $\mathrm{X}_{\mathrm{i}}(\mathrm{m})$ & $\mathrm{Y}_{\mathrm{i}}(\mathrm{m})$ & $\Delta \mathrm{X}_{\mathrm{i}}(\mathrm{m})$ & $\Delta \mathrm{Y}_{\mathrm{i}}(\mathrm{m})$ & $\mathrm{S}_{\mathrm{i}}$ đo $(\mathrm{m})$ & $\Delta_{\mathrm{i}}(\mathrm{m})$ \\
\hline 1 & 2328559.3056 & 581817.9768 & & & & \\
\hline 2 & 2328559.3107 & 581817.9752 & 0.0052 & -0.0016 & 0.0054 & 0.0004 \\
\hline 3 & 2328559.3159 & 581817.9752 & 0.0052 & 0.0000 & 0.0052 & 0.0002 \\
\hline 4 & 2328559.3213 & 581817.9751 & 0.0054 & -0.0002 & 0.0054 & 0.0004 \\
\hline 5 & 2328559.3262 & 581817.9749 & 0.0049 & -0.0002 & 0.0049 & -0.0001 \\
\hline 6 & 2328559.3309 & 581817.9760 & 0.0046 & 0.0011 & 0.0047 & -0.0003 \\
\hline 7 & 2328559.3351 & 581817.9752 & 0.0042 & -0.0008 & 0.0042 & -0.0008 \\
\hline 8 & 2328559.3400 & 581817.9737 & 0.0049 & -0.0015 & 0.0051 & 0.0001 \\
\hline 9 & 2328559.3456 & 581817.9738 & 0.0056 & 0.0000 & 0.0056 & 0.0006 \\
\hline
\end{tabular}

Tài liệu tham khảo

[1]. Đặng Nam Chinh, Đỗ Ngọc Đường (2012), Định vị vệ tinh, Nxb Khoa học Kỹ thuật, Hà Nội

[2]. Hoàng Ngọc Hà, Trương Quang Hiếu (2003), Cơ sở toán hoc xủ lý sồ liệu trắc địa, Nxb Giao thông Vận tải, Hà Nội

\section{Summary}

\section{Evaluate on accuracy of GNSS-RTK technology in some types of engineering surveying}

Tran Viet Tuan, Hanoi University of Mining and Geology

Diem Cong Trang, Vietnam Institute for Building Science and Technology

The content of this article presents the survey results on the accuracy of GNSS-RTK technology in locating single points in Vietnamese conditions, theoretical basis of the empirical measurement survey methods, experimental measurement results and evaluation of this advanced technology applicability in some types of engineering surveying in Vietnam. $\bigcirc$

\section{NGHIÊN CÚ̉U XÂY DỤ゙NG TRƯờNG VẬN TỐC.....}

(Tiếp theo trang 19)

\section{Summary}

\section{Estimation of spatial velocity field in Northern Vietnam by Wavelet Transform}

Lai Van Thuy, Vietnam Institute of Geodesy and Cartography

\section{Duong Chi Cong, Vietnam Association of Geodesy Cartography and Remote Sensing}

The absolute displacement velocity (displacement compares with the center of the Earth Centered Earth Fixed Coordinate System) at points in the geodetic network measured by GNSS shows the displacement of the Earth's crust. Due to the characteristics of geological tectonic activities, they have many unusual and non-linear components. This articale introduces the theoretical basis of the Wavelet Transform method for estimating the spatial velocity field characteristic for Northern Vietnam from GNSS data in period from 2012 to 2015 . Horizontal velocity field has value and direction compatible with ones of Eurasian Plate. Based on vertical velocity field there is any comment on upward and downward motion in studied area. The estimated downward velocities are too large (up to $-2 \mathrm{~cm} / \mathrm{yr}$ ) in North-east and in North and downward (in mountainous region) and upward movements (in South-eastern hollow) here are not really adequate. $\bigcirc$ 